\title{
Interactive Bio-feedback Therapy Using Hybrid Assistive Limbs for Motor Recovery after Stroke: Current Practice and Future Perspectives
}

\author{
Takashi MORISHITA ${ }^{1}$ and Tooru INOUE ${ }^{1}$
}

${ }^{1}$ Department of Neurosurgery, Fukuoka University, Faculty of Medicine, Fukuoka, Japan

\begin{abstract}
Interactive bio-feedback (iBF) was initially developed for the rehabilitation of motor function in patients with neurological disorders, and subsequently yielded the development of the hybrid assistive limb (HAL). Here, we provide a review of the theory underlying HAL treatment as well as our clinical experience and recommendations for future clinical studies using HAL in acute stroke patients. We performed a PubMedbased literature search, a retrospective data review of our acute stroke case series, and included a sample case report of our findings. Given past animal studies and functional imaging results, iBF therapy using the HAL in the acute phase of stroke seems an appropriate approach for preventing learned non-use and interhemispheric excitation imbalances. iBF therapy may furthermore promote appropriate neuronal network reorganization. Based on experiences in our stroke center, HAL rehabilitation is a safe and effective treatment modality for recovering motor impairments after acute stroke, and allows the design of tailored rehabilitation programs for individual patients. iBF therapy through the HAL system seems to be an effective and promising approach to stroke rehabilitation; however, the superiority of this treatment to conventional rehabilitation remains unclear. Further clinical studies are warranted. Additionally, the formation of a patient registry will permit a meta-analysis of HAL cases and address the problems associated with a controlled trial (e.g., the heterogeneity of an acute stroke cohort). The development of robotic engineering will improve the efficacy of HAL rehabilitation and has the potential to standardize patient rehabilitation practice.
\end{abstract}

Key words: hybrid assistive limb, interactive bio-feedback, cybernetics, stroke, neurorehabilitation

\section{Introduction}

Stroke is a vascular disease that produces severe sequelae including motor impairment, aphasia, and cognitive problems. The early initiation of highquality rehabilitation is vital for the preservation and recovery of brain functions., ${ }^{1,2}$ Among various treatment modalities, robot rehabilitation has attracted increasing attention in the field of stroke due to recent reports of its efficacy. ${ }^{3,4)}$ In particular, the advent of the hybrid assistive limb (HAL; Cyberdyne Inc., Tsukuba, Japan) has potential to change the rehabilitative approach to stroke.

Robot rehabilitation is a growing field supported by various studies. The recently published stroke rehabilitation guidelines from the American Heart Association/American Stroke Association mentioned

Received March 22, 2016; Accepted July 22, 2016 "robot-assisted movement training to improve motor function and mobility after stroke in combination with conventional therapy may be considered." ${ }^{5}$ Among various robots, HAL is a unique exoskeletontype robot suit that was developed by Sankai and colleagues for neurorehabilitation based on the "interactive bio-feedback (iBF)" theory. ${ }^{5,6)}$ The HAL is designed to support the paretic limb by detecting bioelectrical signals (BES) to predict and assist muscle movement. This system makes the HAL robot unique among various rehabilitation robots as most robotic ambulation trainers allow passive movements for patients. HAL robots, however, allow patients to move limbs more voluntarily than other robots. Currently, three types of HAL robots are available for rehabilitation: a bilateral leg type (BL), a single leg type (SL), and a single joint type (SJ), ${ }^{6,7)}$

The HAL has been widely applied for various neurological disorders and shown to be effective to 
facilitate the functional recovery. The HAL therapy has been approved for medical use for patients with gait disability following spinal cord injury in Germany since 2013. Additionally, it should be noted that the use of the HAL was approved for national insurance coverage to treat rare neurological disorders based on the excellent outcomes of a clinical trial (study NCY-3001, JMACCT ID: JMA-ILA00156) in Japan. These rare neurological disorders include spinal muscular atrophy, spinal and bulbar muscular atrophy, amyotrophic lateral sclerosis, Charcot-Marie-Tooth disease, distal myopathy, inclusion body myositis, congenital mypathy, and muscular dystrophy.

In this paper, we will briefly review the theories supporting HAL-assisted rehabilitation and examine the available clinical data regarding its utility. We will then discuss strategies to improve the stroke rehabilitation paradigm and provide recommendations for future clinical studies using HAL in acute stroke.

\section{Methods}

We performed a PubMed-based literature review of the theories underlying HAL-supported rehabilitation. We also performed a retrospective data review of 141 patients in total who underwent rehabilitation therapy using HAL, and describe our implementation of HAL for the rehabilitation of acute stroke at Fukuoka University Hospital.

\section{Results}

\section{Stroke rehabilitation and interactive bio- feedback theory}

Stroke-related disabilities are determined by the degree and location of the stroke lesion, such that medical and surgical interventions in the acute phase seek to minimize damage to the brain. Motor impairment following stroke is thought to result from lesions of the cortico-spinal tract (CST), and the preservation of motor performance depends on the integrity of the CST (Fig. 1). ${ }^{7,8)}$ Indeed, recent studies have shown that a cascade of maladaptive neuronal network reorganization resulting in permanent neurological deficits occurs in the hyper-acute phase of stroke due to damage to the central nervous system (CNS). ${ }^{8,9)}$

One factor thought to prevent motor recovery following stroke is explained by the "learned non-use" hypothesis, which is a basic theory of constraintinduced therapy. ${ }^{10,11)}$ This theory hypothesizes that a failure to perform successful movements with the paretic limb makes the patient less motivated to use that limb and facilitates overuse of the non-paretic limb. Therefore, use-dependent and rehabilitative neuroplastic changes in the brain are prevented..$^{9,10)}$
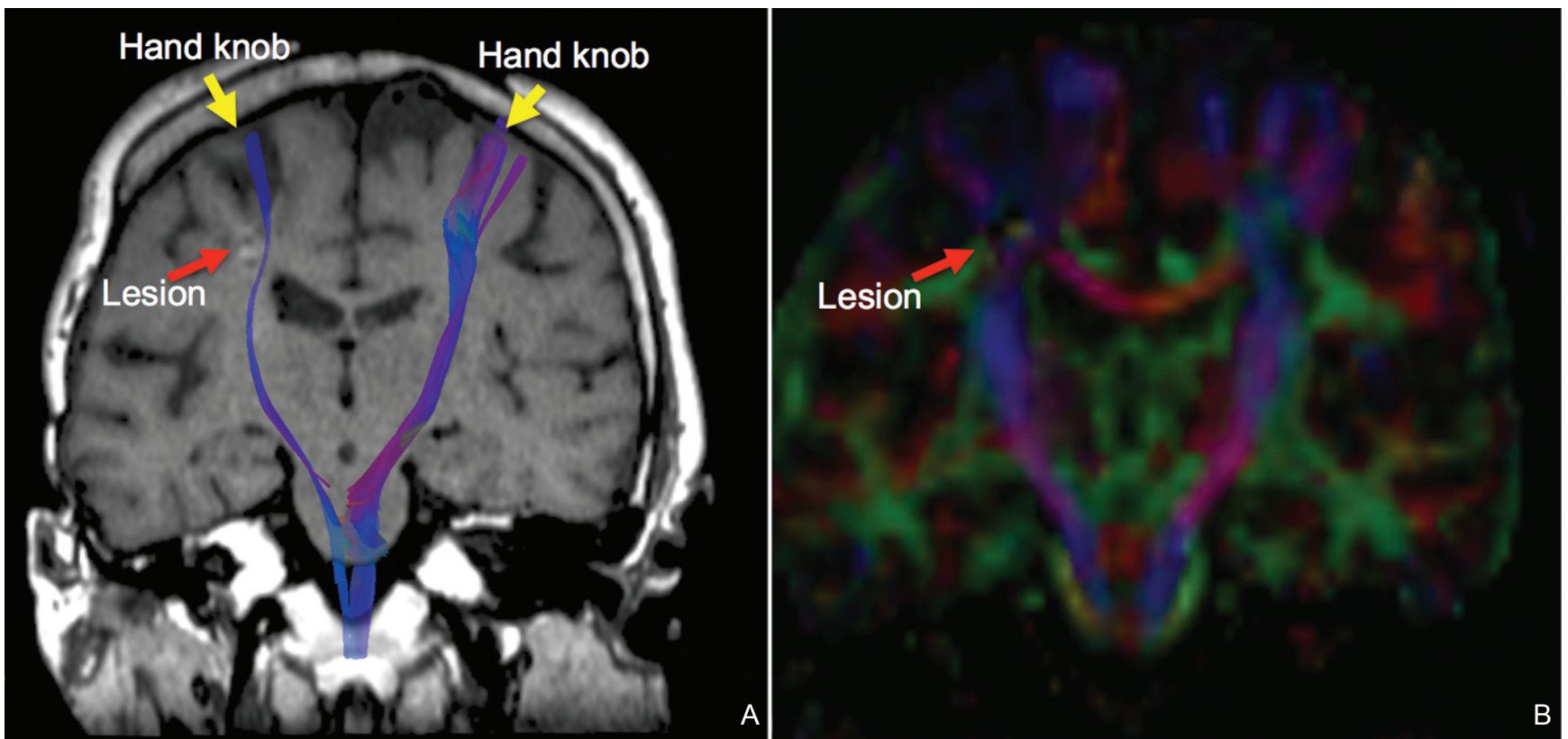

Fig. 1 Coronal MRI images of a post-stroke patient. A: Fiber tractography superimposed onto a $T_{1}$-weighted image. Fiber tract integrity was diminished on the right side due an ischemic lesion. B: A coronal fractional anisotropy mapping image showing the lesion in the right corona radiata. 
Another factor preventing motor recovery relates to an interhemispheric imbalance of excitability due to maladaptive compensatory changes in the contralesional hemisphere. ${ }^{9-13)}$ Increased excitability in the contralesional somatosensory cortex has been demonstrated following the induction of small ischemic lesions in several animal studies in acute and chronic stroke. ${ }^{13,14)}$ In addition, recent functional magnetic resonance imaging (fMRI) studies have reported hyperactivity of the contralesional hemisphere after stroke, and additionally suggested that the hyperactive contralesional hemisphere might inhibit the activities of the lesional hemisphere. ${ }^{11,12)}$ Another fMRI study also showed increased functional connectivity between the bilateral primary motor cortices following stroke. ${ }^{15,16)}$ Interhemispheric imbalances may be aggravated by non-use of the paretic limb as well. ${ }^{9,10)}$

The concept of iBF theory was first proposed by Sankai and colleagues to promote functional recovery from neurological disorders. The iBF theory was initially tested in animal studies, ${ }^{16,17)}$ and the HAL exoskeleton robot was developed as an implementation of iBF theory in humans., ${ }^{5,6)}$ According to the theory, the motor signals are generated in the CNS and conducted via peripheral nerves to initiate muscle activity; these signals detected as BES then trigger the motion of the HAL supporting the paretic limb (interaction). Sensory input is then sent back to the CNS to activate the impaired neuronal networks (bio-feedback) and the activated CNS in turn enhances its motor output. The formation of this closed loop is considered to promote the neuroplasticity and facilitate the recovery from stroke-related CNS damage (Fig. 2). Therefore, HAL therapy is an appropriate approach to break the vicious cycle of the maladapted neuronal remodeling related to non-use of the paretic limb and subsequent interhemispheric imbalance.

\section{Paradigm shift of HAL rehabilitation}

The first HAL robot designed to provide bilateral leg support (HAL-BL) was introduced to our hospital in September of 2011. The single-leg model of HAL (HAL-SL) was subsequently introduced in November of 2011, and we began using two types of single-joint HAL (HAL-SJ) for elbow and knee joint therapy in October of 2013.

We used HAL-assisted rehabilitation in 141 cases (97 lower extremity cases and 45 upper extremity
A

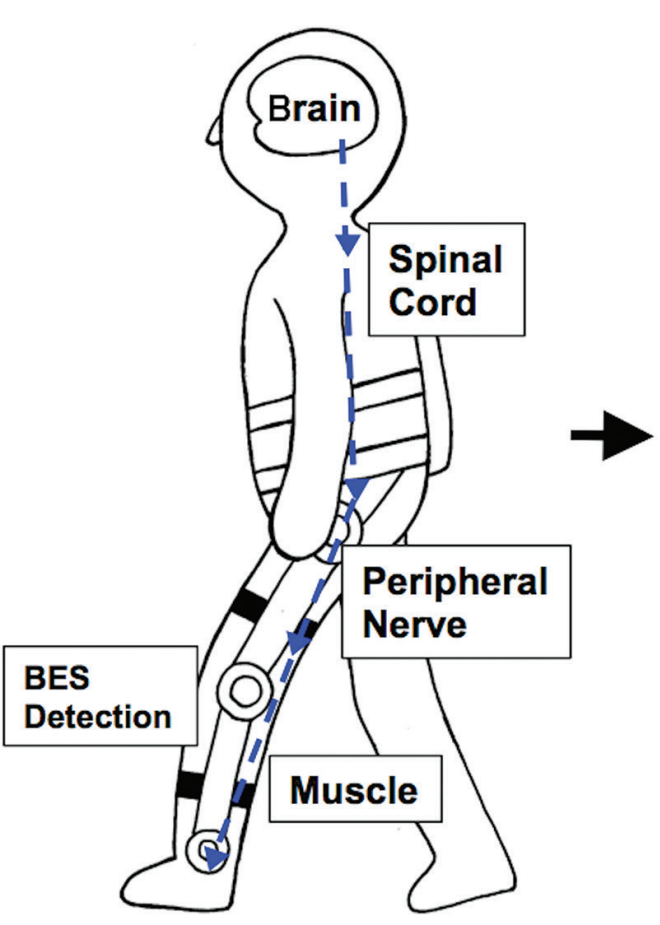

B

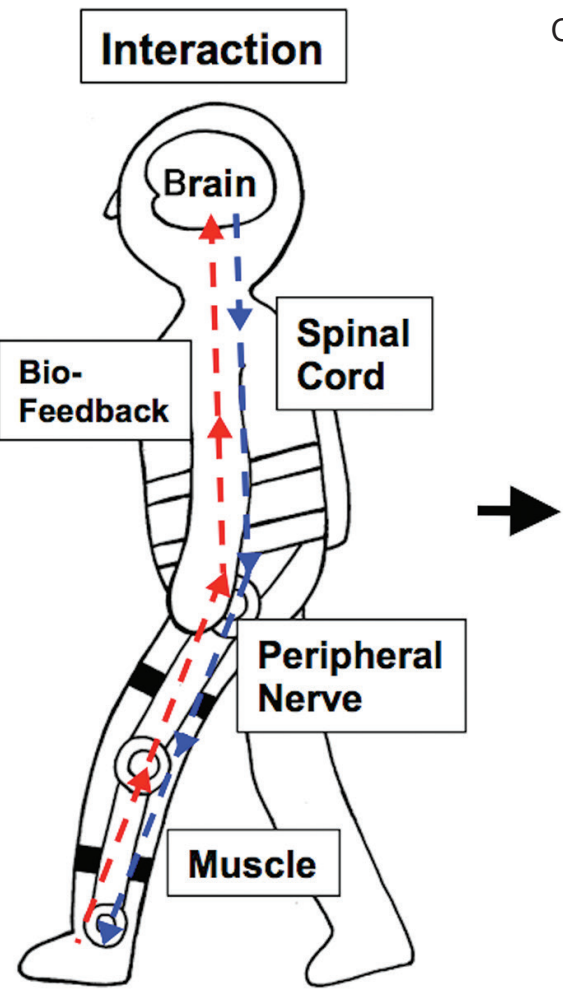

C

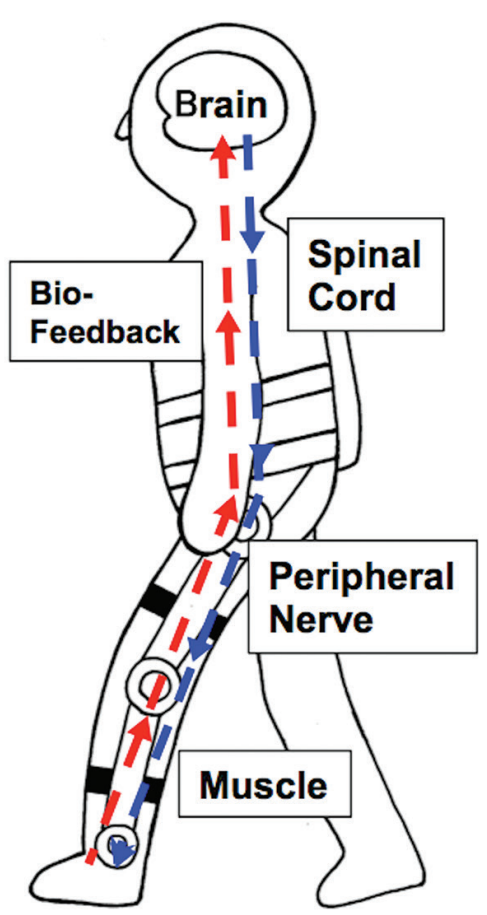

Fig. 2 Conceptualization of the closed loop system formed by interactive bio-feedback. A) Bioelectrical signal from the impaired corticospinal tract is detected and the voluntary muscle movement is assisted by HAL. B) Then, the sensory signal is sent back to the brain. C) Brain-machine interaction strengthens the signal from the corticospinal tract. 
cases) of acute stroke out of a total of 247 cases of neurological disorders at our center between November 2011 and February 2016. In the early series (from November 2011 to March 2012), we attempted to use HAL in every case regardless the severity of cognitive or motor deficit in order to test feasibility. The first feasibility study evaluated the risks associated with HAL-supported rehabilitation in 22 acute stroke cases using the HAL-BL. ${ }^{17,18)}$ The same study demonstrated that intracerebral hemorrhage cases and severe hemiplegic cases with lower Brunnstrom stages were at higher risk of orthostatic hypotension, despite the fact that HAL therapy was safely performed in general. ${ }^{17,18)}$ Of note, six patients refused to continue HAL therapy after the initial rehabilitation session of this study.

We next retrospectively reviewed the clinical data of 53 acute stroke patients who underwent neurorehabilitation using either the HAL-BL or HAL-SL between November 2011 and May 2014 to determine the cases where HAL treatment was effective for ambulatory training. ${ }^{18,19)}$ In this previous report, we classified the included patients according to Brunnstrom stage severity and evaluated the activities of daily living (ADL) and motor performance. For patients Brunnstrom stage III or higher, improvements were seen in the Barthel index, functional independence measure (FIM), and the 10-m walking test (10MWT).

We also reviewed the long-term outcomes of HAL treatment in supratentorial hemorrhagic stroke. ${ }^{19,20)}$ In this study, clinical outcomes at approximately 4 months after stroke were assessed in a HAL rehabilitation group (14 cases; 11 of which continued HAL rehabilitation through the follow-up period) and a non-HAL rehabilitation group (77 cases). Though no significant differences were observed between the overall outcomes of the two groups, HAL rehabilitation produced significant improvements in the FIM scores of patients with right-sided lesions.

The advent of the HAL-SJ has also enabled us to treat motor weakness of the upper extremities and severe cases at bedside. As the severity and phenomenology of acute stroke are heterogeneous, we consider that rehabilitation approaches should be tailored for each case. The availability of a variety of machines offers the advantageous option for therapists to select an appropriate treatment modality and tailor the treatment plan for each patient. Most recently, we observed that tailored rehabilitation using multiple HAL robot types produced significant improvements in ADL scores compared with the single robot treatment. ${ }^{6}$ It should be also noted that the early initiation of iBF therapy for both lower and upper extremity impairments is desirable for the facilitation of functional recovery in terms of neuronal network remodeling.

\section{Current rehabilitation protocol}

Here, we will briefly describe how we have tailored rehabilitation strategies using multiple robots. Concerning gait disability, we consider that there are three stages of motor impairment. First, the axial function of the trunk is important for the maintenance of a seated or upright posture. For lower extremity training, we start with the HAL-SJ at bedside to facilitate knee joint movement prior to ambulation training. Once the patient achieves the sitting position, we begin using HAL-BL for gait training. As the HAL-BL supports both legs, the patient is able to learn by feel how the robot supports the paretic limb by moving the non-paretic limb. In addition to ambulation training, the patient practices the extension and flexion of the paretic leg in a seated position, and repeats the exercise standing up and sitting down. Finally, for upper extremity training, elbow extension and flexion exercises are repeated 100-150 times at each session.

\section{Representative case}

Though no published data has demonstrated the facilitative effect of HAL therapy, we consider that HAL rehabilitation provides an advantageous acceleration of functional recovery after acute stroke. Here, we present a case where HAL rehabilitation successfully facilitated motor recovery. The patient was a 67-year-old woman who was admitted to our emergency department complaining of left hemiparesis due to right frontal lobar hemorrhage (Fig. 3A). Her past medical history included breast cancer, so she underwent endoscopic evacuation of the hemorrhage for diagnostic treatment, which was negative for malignant cells. The patient was subsequently managed in our stroke care unit. Rehabilitation was initiated on postoperative day 3 using the HAL-SJ (Fig. 3B). The baseline Brunnstrom stage of the left upper extremity was III on postoperative day 3 , and improved to stage IV after nine rehabilitation sessions using the HAL-SJ. The patient's Fugl-Meyer assessment score of the left upper extremity also improved from 5 to 23 after rehabilitation. Ambulation training was initiated on the postoperative day 11 with the HAL-BL and dramatic gait improvements were observed after each session: prior to the first HAL session, the patient's 10MWT results were 14 seconds with 21 steps, which improved to 12 seconds with 20 steps immediately after the first ambulation training session. After two sessions with the HAL-BL (Fig. 3C), the patient underwent two sessions with the HAL-SL (Fig. 3D). After these 


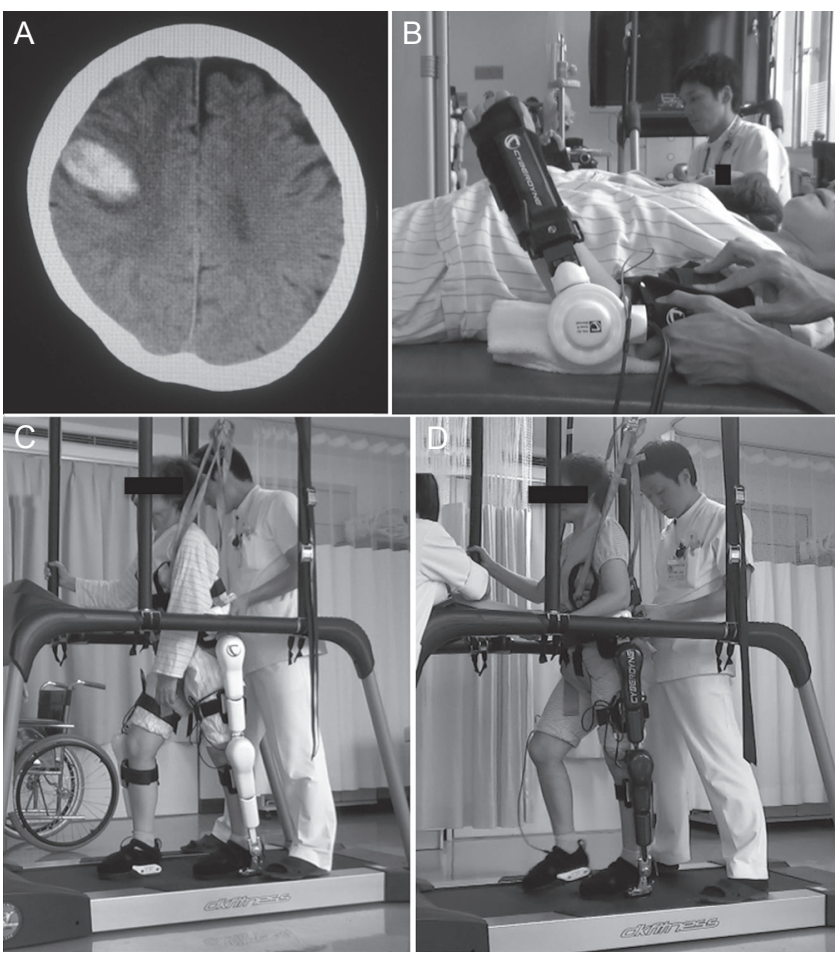

Fig. 3 CT scan image and overview of rehabilitation of the representative case. A) A CT image showing right frontal lobar hemorrhage in the representative case. B) HAL-SJ for upper extremity training. C) Bilateral leg version of HAL. D) Single leg version of HAL.

sessions on postoperative day 21 , the patient's 10MWT results were 9 seconds with 17 steps. She was transferred to a rehabilitation facility on postoperative day 21 for further treatment. This case illustrates the facilitative effect of HAL treatment on motor functional recovery after stroke.

\section{Discussion}

In this paper, we have reviewed theories regarding the mechanism by which HAL potentially modulates neuronal networks in stroke patients and presented our own clinical experiences using HAL robots in the rehabilitation of acute stroke patients. iBF therapy through the use of the HAL system seems to be an effective and promising treatment modality for select cases. However, only a few clinical studies of acute stroke rehabilitation have used HAL and reported favorable outcomes, other than our own reports. ${ }^{21,22)}$ In fact, the clinical evidences currently available for the use of HAL after acute stroke have all been case series with the exception of one paper from our group. ${ }^{19,20)}$ Thus, it remains difficult to conclude that the HAL system is superior to conventional rehabilitation therapies. Randomized controlled trials with larger samples are warranted.

To maximize the clinical outcomes of HAL rehabilitation, patient selection is important. ${ }^{18,23)}$ Similar to conventional rehabilitation therapies, intact cognitive function is important for treatment efficacy as the patient is required to follow commands given by the therapist. A recent report emphasized the importance of evaluating cognitive function prior to the initiation of HAL rehabilitation from the standing point of HAL suitability as defined by clinical efficacy. ${ }^{23)}$ Secondly, it should be noted that patients with complete paralysis are unable to use the HAL system as the HAL requires BES generated by voluntary muscle movement. To this end, patients with severe motor weakness (Brunnstrom stages I and II) may also be inappropriate candidates for HAL rehabilitation therapy. ${ }^{18,19)}$ Additionally, it should be noted as a limitation that even though the advantage of robot rehabilitation is considered to reduce the burden of therapists, at least two therapists are needed to use HAL for ambulation training.

In future, clinical studies evaluating the efficacy of HAL rehabilitation therapy after acute stroke, we advocate the formation of a registry to enable meta-analyses and various subanalyses of the clinical data. In this registry, a variety of clinical information should be recorded using universal clinical assessment batteries. The baseline characteristics should, at minimum, include the age at stroke onset, sex, handedness, location of the stroke lesion, stroke type (ischemic versus hemorrhagic), Brunnstrom stage, National Institutes of Health Stroke Scale score, and Modified Rankin Scale score. For pre- versus post-intervention analyses, motor function, ADL, and quality of life should be evaluated. Baseline characteristics and clinical outcome measures that should be included in the HAL registry are summarized in Table 1.

In addition to the above-mentioned clinical evaluations, more detailed imaging studies including fMRI, diffusion tensor imaging, and near infrared spectroscopy (NIRS) should be considered depending on their availability at academic centers. In particular, NIRS has drawn attention as a unique imaging modality that can provide real-time information to the patient in a manner that enables bio-feedback. ${ }^{24,25)}$ These imaging modalities may also shed light on the mechanisms of action of HAL rehabilitation therapy and provide more robust evidence for its clinical efficacy from a basic science standpoint.

As neurorehabilitation is a developing field, there have been a number of emerging treatment modalities including brain stimulation therapy, ${ }^{20,26,27)}$ virtual reality training, electromyography biofeedback, and so on. ${ }^{5)}$ Among these treatment modalities, HAL 
Table 1 Recommended clinical outcome measures for patients undergoing HAL rehabilitation

\begin{tabular}{|c|c|}
\hline & Evaluation measures \\
\hline $\begin{array}{l}\text { Patient } \\
\text { demographics }\end{array}$ & $\begin{array}{l}\text { Age, sex, handedness, stroke etiology } \\
\text { (i.e. ischemic vs hemorrhagic, location } \\
\text { of stroke lesion, etc.), primary stroke } \\
\text { treatment (i.e. medications, surgical } \\
\text { procedures, etc.), starting date of } \\
\text { rehabilitation, duration of HAL } \\
\text { treatment, number of HAL treatment } \\
\text { sessions }\end{array}$ \\
\hline $\begin{array}{l}\text { Stroke } \\
\text { severity } \\
\text { assessment }\end{array}$ & $\begin{array}{l}\text { Glasgow coma scale (GCS), modified } \\
\text { rankin scale (mRS), national institute } \\
\text { of health stroke scale (NIHSS), stroke } \\
\text { impairment assessment set (SIAS) }\end{array}$ \\
\hline $\begin{array}{l}\text { Motor } \\
\text { function }\end{array}$ & $\begin{array}{l}\text { Fugl-Meyer assessment (FMA), 10-m } \\
\text { walking test (10MWT), 2-minute walk } \\
\text { test, action research arm test (ARAT), } \\
\text { Berg balance scale (BBS) }\end{array}$ \\
\hline $\begin{array}{l}\text { Activities of } \\
\text { daily living }\end{array}$ & $\begin{array}{l}\text { Barthel index (BI), functional } \\
\text { independence measure (FIM) }\end{array}$ \\
\hline Quality of life & SF-36 \\
\hline Cognition & $\begin{array}{l}\text { Mini-mental status exam (MMSE), } \\
\text { Motreal cognitive assessment (MoCA) }\end{array}$ \\
\hline Mood & $\begin{array}{l}\text { Apathy scale, self-rating depression } \\
\text { scale (SDS) }\end{array}$ \\
\hline Others & $\begin{array}{l}\text { Modified Ashworth scale (for spasticity } \\
\text { assessment), adverse events (for safety } \\
\text { assessment), imaging studies including } \\
\text { fMRI, DTI, and NIRS }\end{array}$ \\
\hline
\end{tabular}

DTI: diffusion tensor imaging, fMRI: functional magnetic resonance imaging, NIRS: near infrared spectroscopy.

therapy based on iBF theory is unique in the point that the robot strengthens the residual voluntary movements by an interaction between the robot and the patient rather than regaining completely lost abilities. Additionally, we have recently published three cases where HAL treatment was effective for ataxic gait due to acute stroke, and this may be a strength of this robot rehabilitation which other robotic treatments do not possess. ${ }^{28)}$ Clinicians should be aware that the each treatment modality has advantages and disadvantages and thus the treatment should be tailored for each patient.

\section{Conclusions}

At present, it is difficult to conclude that HALassisted rehabilitation is superior to conventional rehabilitation therapies due to a paucity of evidence; however, iBF-based therapy using the HAL seems to be a promising treatment option for rehabilitation after stroke. Further clinical studies are warranted to demonstrate the efficacy of this new therapy. The formation of a multi-center registry for stroke cases managed with HAL rehabilitation will help improve our understanding of its mechanisms of action and clinical outcomes.

Of note, clinicians should be aware that there is a critical difference between manual and robot rehabilitation. While the clinical outcomes of manual rehabilitation can be determined by the therapists' own experience and skills, those of robot rehabilitation are determined by the performance ability of the robot. As robot engineering improves, and the current weaknesses associated with robot rehabilitation may be addressed. We, therefore, believe that the future of stroke practice will be improved and standardized with the development of the HAL system.

\section{Acknowledgements}

The authors are grateful for the clinical work of our neurorehabilitation team: Dr. Etsuji Shiota, M.D., Ph.D., Dr. Hiroyuki Fukuda, P.T., Ph.D., Mr. Kazuya Saita, O.T., Mr. Hyakutake, O.T., Ms. Junko Watanabe, S.T., and Ms. Aya Yatsugi, P.T. This study was in part supported by a Japan Society for the Promotion of Science Grant-in-Aid for young scientists (B) 15K19984, the Takeda Science Foundation, the Uehara Memorial Foundation, Central Research Institute of Fukuoka University (No. 161042), and the Clinical Research Promotion Foundation in Japan. We also appreciate Ms. Asuka Ikezaki for her illustration (Figure 2).

\section{Conflicts of Interest Disclosure}

The authors have no conflicts of interest to report.

\section{References}

1) Jauch EC, Saver JL, Adams HP, Jr., Bruno A, Connors JJ, Demaerschalk BM, Khatri P, McMullan PW, Qureshi AI, Rosenfield K, Scott PA, Summers DR, Wang DZ, Wintermark M, Yonas H; American Heart Association Stroke Council; Council on Cardiovascular Nursing; Council on Peripheral Vascular Disease; Council on Clinical Cardiology: Guidelines for the early management of patients with acute ischemic stroke: a guideline for healthcare professionals from the American Heart Association/American Stroke Association. Stroke 44: 870-947, 2013

2) Morgenstern LB, Hemphill JC, 3rd, Anderson C, Becker K, Broderick JP, Connolly ES, Jr., Greenberg SM, Huang JN, MacDonald RL, Messé SR, Mitchell PH, Selim M, Tamargo RJ; American Heart Association Stroke Council and Council on Cardiovascular 
Nursing: Guidelines for the management of spontaneous intracerebral hemorrhage: a guideline for healthcare professionals from the American Heart Association/American Stroke Association. Stroke 41: 2108-2129, 2010

3) Basteris A, Nijenhuis SM, Stienen AH, Buurke JH, Prange GB, Amirabdollahian F: Training modalities in robot-mediated upper limb rehabilitation in stroke: a framework for classification based on a systematic review. J Neuroeng Rehabil 11: 111, 2014

4) Norouzi-Gheidari N, Archambault PS, Fung J: Effects of robot-assisted therapy on stroke rehabilitation in upper limbs: systematic review and meta-analysis of the literature. J Rehabil Res Dev 49: 479-496, 2012

5) Winstein CJ, Stein J, Arena R, Bates B, Cherney LR, Cramer SC, Deruyter F, Eng JJ, Fisher B, Harvey RL, Lang CE, MacKay-Lyons M, Ottenbacher KJ, Pugh S, Reeves MJ, Richards LG, Stiers W, Zorowitz RD; American Heart Association Stroke Council, Council on Cardiovascular and Stroke Nursing, Council on Clinical Cardiology, and Council on Quality of Care and Outcomes Research: Guidelines for adult stroke rehabilitation and recovery: a guideline for healthcare professionals from the American Heart Association/American Stroke Association. Stroke 47: e98-e169, 2016

6) Suzuki K, Mito G, Kawamoto H, Hasegawa Y, Sankai Y: Intention-based walking support for paraplegia patients with robot suit HAL. Adv Robotics 21: 1441-1469, 2007

7) Fukuda H, Morishita T, Ogata T, Saita K, Hyakutake K, Watanabe J, Shiota E, Inoue T: Tailor-made rehabilitation approach using multiple types of hybrid assistive limb robots for acute stroke patients: A Pilot Study. Assist Technol 28: 53-56, 2016

8) Stinear CM, Barber PA, Smale PR, Coxon JP, Fleming MK, Byblow WD: Functional potential in chronic stroke patients depends on corticospinal tract integrity. Brain 130: 170-180, 2007

9) Wahl AS, Schwab ME: Finding an optimal rehabilitation paradigm after stroke: enhancing fiber growth and training of the brain at the right moment. Front Hum Neurosci 8: 381, 2014

10) Xerri C, Zennou-Azogui Y, Sadlaoud K, Sauvajon D: Interplay between intra- and interhemispheric remodeling of neural networks as a substrate of functional recovery after stroke: adaptive versus maladaptive reorganization. Neuroscience 283: 178-201, 2014

11) Taub E, Uswatte G, Mark VW, Morris DM: The learned nonuse phenomenon: implications for rehabilitation. Eura Medicophys 42: 241-256, 2006

12) Grefkes C, Fink GR: Connectivity-based approaches in stroke and recovery of function. Lancet Neurol 13: 206-216, 2014

13) Volz LJ, Sarfeld AS, Diekhoff S, Rehme AK, Pool EM, Eickhoff SB, Fink GR, Grefkes C: Motor cortex excitability and connectivity in chronic stroke: a multimodal model of functional reorganization. Brain Struct Funct 220: 1093-1107, 2015

14) Mohajerani MH, Aminoltejari K, Murphy TH: Targeted mini-strokes produce changes in interhemispheric sensory signal processing that are indicative of disinhibition within minutes. Proc Natl Acad Sci USA 108: E183-E191, 2011

15) Takatsuru Y, Fukumoto D, Yoshitomo M, Nemoto T, Tsukada H, Nabekura J: Neuronal circuit remodeling in the contralateral cortical hemisphere during functional recovery from cerebral infarction. J Neurosci 29: 10081-10086, 2009

16) Liu J, Qin W, Zhang J, Zhang X, Yu C: Enhanced interhemispheric functional connectivity compensates for anatomical connection damages in subcortical stroke. Stroke 46: 1045-1051, 2015

17) Sankai Y, Fujiwara K, Watanabe K, Moriyama $H$ : HOJO-brain for motion control of robots and biological systems. Artif Life Robotics 2: 162-169, 1998

18) Ueba $T$, Hamada O, Ogata $T$, Inoue $T$, Shiota $E$, Sankai Y: Feasibility and safety of acute phase rehabilitation after stroke using the hybrid assistive limb robot suit. Neurol Med Chir (Tokyo) 53: 287-290, 2013

19) Fukuda H, Samura K, Hamada O, Saita K, Ogata T, Shiota E, Sankai Y, Inoue T: Effectiveness of acute phase hybrid assistive limb rehabilitation in stroke patients classified by paralysis severity. Neurol Med Chir (Tokyo) 55: 487-492, 2015

20) Ogata T, Abe H, Samura K, Hamada O, Nonaka M, Iwaasa M, Higashi T, Fukuda H, Shiota E, Tsuboi Y, Inoue T: Hybrid assistive limb (hal) rehabilitation in patients with acute hemorrhagic stroke. Neurol Med Chir (Tokyo) 55: 901-906, 2015

21) Maeshima S, Osawa A, Nishio D, Hirano Y, Takeda K, Kigawa H, Sankai Y: Efficacy of a hybrid assistive limb in post-stroke hemiplegic patients: a preliminary report. BMC Neurol 11: 116, 2011

22) Nilsson A, Vreede KS, Häglund V, Kawamoto H, Sankai Y, Borg J: Gait training early after stroke with a new exoskeleton-the hybrid assistive limb: a study of safety and feasibility. J Neuroeng Rehabil 11: 92, 2014

23) Chihara H, Takagi Y, Nishino K, Yoshida K, Arakawa Y, Kikuchi T, Takenobu Y, Miyamoto S: Factors predicting the effects of hybrid assistive limb robot suit during the acute phase of central nervous system injury. Neurol Med Chir (Tokyo) 56: 33-37, 2016

24) Mihara $M$, Hattori $N$, Hatakenaka M, Yagura $H$, Kawano T, Hino T, Miyai I. Near-infrared spectroscopy-mediated neurofeedback enhances efficacy of motor imagery-based training in poststroke victims: a pilot study. Stroke 44: 1091-1098, 2013

25) Obrig H: NIRS in clinical neurology - a 'promising' tool? Neuroimage 85: 535-546, 2014

26) Morishita $T$, Inoue $T$. Brain stimulation therapy for central post-stroke pain from a perspective of 
interhemispheric neural network remodeling: Front Hum Neurosci 10: 166, 2016

27) Morishita T, Hyakutake K, Saita K, Takahara M, Shiota E, Inoue T: Pain reduction associated with improved functional interhemispheric balance following transcranial direct current stimulation for post-stroke central pain: A case study. J Neurol Sci 358: 484-485, 2015

28) Hamada O, Samura K, Abe H, Fukuda H, Ogata T, Nonaka M, Higashi T, Shiota E, Inoue T: Three cases with ataxic gait disorder improved by using a hybrid assistive limb robot suit. Jpn J Neurosurg 24: 413-419, 2015

Address reprint requests to: Takashi Morishita, MD, PhD, Department of Neurosurgery, Fukuoka University Faculty of Medicine, Nanakuma 7-45-1, Jonan ward, Fukuoka, Japan.

e-mail: tmorishita@fukuoka-u.ac.jp 